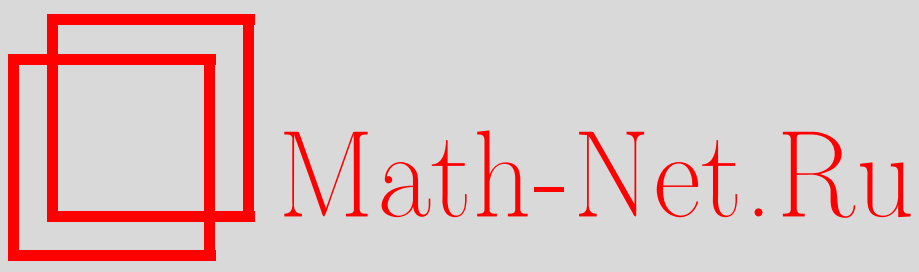

G. G. Gevorkyan, M. G. Grigoryan, Absolute convergence of the double fourier-franklin series, Sibirsk. Mat. Zh., 2020, Volume 61, Number 3, 513-527

DOI: https://doi.org/10.33048/smzh.2020.61.303

Use of the all-Russian mathematical portal Math-Net.Ru implies that you have read and agreed to these terms of use http://www . mathnet.ru/eng/agreement

Download details:

IP : 54.205 .225 .156

April 26, 2023, 17:06:56

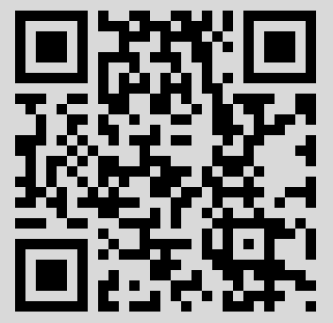


Сибирский математический журнал Май-июнь, 2020. Том 61, № 3

УДК 517.51

\title{
АБСОЛЮТНАЯ СХОДИМОСТЬ ДВОЙНЫХ РЯДОВ ФУРЬЕ — ФРАНКЛИНА
}

\section{Г. Г. Геворкян, М. Г. Григорян}

\begin{abstract}
Аннотация. Доказано, что для любого $0<\epsilon<1$ существует измеримое множество $E \subset T=[0,1]^{2}$ с мерой $|E|>1-\epsilon$ такое, что для каждой функции $f \in L^{1}(T)$ и для любого $0<\eta<1$ можно найти функцию $\tilde{f} \in L^{1}(T)$ с $\iint_{T}|f(x, y)-\tilde{f}(x, y)| d x d y \leq \eta$, совпадающую с $f(x, y)$ на $E$ и такую, что ее двойной ряд Фурье - Франклина абсолютно сходится к ней почти всюду на $T$.
\end{abstract}

DOI 10.33048/smzh.2020.61.303

Ключевые слова: двойные ряды Фурье, система Франклина, абсолютная сходимость.

\section{$\S 1$. Введение}

Статья посвящена изучению абсолютной сходимости почти всюду рядов по двойной системе Франклина с точки зрения классических теорем Лузина [1] и Меньшова [2] «об исправлении функций».

Система Франклина [3] является одной из популярных систем функций, ее изучению посвящено много работ. Одно из главных свойств этой системы состоит в том, что она образует ортогональный базис в пространстве $C[0,1]$ и безусловный базис в пространствах $L^{p}[0,1], p \in(1, \infty), C[0,1]-$ класс всех непрерывных на $[0,1]$ функций $\left(\|f\|_{C}=\max _{x \in[0,1]}|f(x)|\right), L^{p}[0,1](p>0)$ - класс измеримых на $[0,1]$ функций, для которых $\int|f(x)|^{p} d x<\infty$. Меру Лебега множества $E \subseteq[0,1]\left(E \subseteq T=[0,1]^{2}\right)$ будем обозначать через $|E|$.

Вопросам сходимости рядов Фурье по классическим системам после изменения значений функции на множестве малой меры посвящено много работ.

Широко известен следующий результат.

Теорема А [2]. Пусть $f(x)$ - измеримая функция, конечная почти всюду на $[0,2 \pi]$. Каково бы ни было $\epsilon>0$, можно определить непрерывную функцию $g(x)$, совпадающую $c f(x)$ на некотором множестве $E$ с мерой $|E|>2 \pi-\epsilon$ и такую, что ее ряд Фурье по тригонометрической системе сходится равномерно на $[0,2 \pi]$.

Далее в этом направлении получено много интересных результатов.

Приведем те результаты, которые непосредственно относятся к результатам настоящей работы.

Исследование выполнено при финансовой поддержке ГКН МОН РА в рамках научных проектов 18-1А074 и 18-1А148.

(c) 2020 Геворкян Г. Г., Григорян М. Г. 
Теорема Б [4]. Для любой измеримой почти всюду конечной на [0, 1] функции $f(x)$ и для любого числа $0<\epsilon<1$ можно найти функцию $\tilde{f} \in L^{2}[0,1) c$ $|\{x: f(x) \neq \tilde{f}(x)\}|<\epsilon$ так, что ее ряд Фурье по системе Хаара абсолютно сходится равномерно на $[0,1]$.

Теорема В [5]. Для любой непрерывной на [0,1] функции $f(x)$ и для любого числа $0<\epsilon<1$ можно найти функцию $\tilde{f} \in C[0,1] c|\{x: f(x) \neq \tilde{f}(x)\}|<\epsilon$ так, что ее ряд Фурье по системе Франклина абсолютно сходится равномерно на $[0,1]$.

Следует отметить, что Кацнельсон [6] доказал, что в теореме Меньшова невозможно добиться абсолютной сходимости (т. е. теорема В для тригонометрической системы неверна).

Теорема $\Gamma$ [7]. Для любого $0<\epsilon<1$ существует измеримое множество $E \subset[0,1]$ с мерой $|E|>1-\epsilon$ такое, что для каждой функции $f \in L^{1}[0,1)$ можно найти функцию $\tilde{f} \in L^{1}[0,1)$, совпадающую $c f$ на $E$ и такую, что ее ряд Фурье по системе Хаара абсолютно сходится почти всюду на $[0,1]$ и все ненулевые члены в последовательности коэффициентов Фурье вновь полученной функции по системе Хаара расположены в убывающем порядке.

Отметим, что «исключительное» множество $e$, на котором происходит изменение функции $f(x)$, в теореме $Г$ универсально (обслуживает целый функциональный класс), а в теоремах Б и В существенно зависит от исправляемой функции $f(x)$, в этих теоремах множество $e$ невозможно выбрать не зависящим от функции $f(x)$. Этот факт для системы Франклина вытекает из следующей теоремы, доказанной в [8].

Теорема Д. Для любого множества $E$ положительной меры и для каждой его точки плотности $x_{0}$ существует непрерывная функция $f_{0} \in C[0,1]$ такая, что для любой ограниченной функции $f(x)$, совпадающей $c$ функцией $f_{0}(x)$ на множестве $E$, ряд Фурье - Франклина функции $f(x)$ абсолютно расходится в точке $x_{0}$.

Эта теорема верна и для системы Хаара. Отметим, что это «плохое» свойство не является общим для всех базисов пространства $C[0,1]$, в частности, в [9] доказано, что система Фабера - Шаудера не обладает этим свойством. А именно, имеет место

Теорема Е. Для любого $0<\epsilon<1$ существует измеримое множество $E \subset$ $[0,1]$ с мерой $|E|>1-\epsilon$ такое, что для каждой функции $f \in C[0,1]$ можно найти функцию $g \in C[0,1]$, совпадающую $c f$ на $E$ и такую, что ее разложение $\sum_{k=0}^{\infty} A(g) \varphi_{k}(x)$ по системе Фабера - Шаудера абсолютно сходится равномерно на $[0,1]$ и

$$
\left\|\sum_{n=1}^{\infty}\left|A_{n}(g)\right| \varphi_{n}\right\|_{C} \leq\|g\|_{C}<2\|f\|_{C} .
$$

Сразу возникает вопрос, ответ на который нам не известен.

Вопрос 1. Существует ли ортогональный базис пространства $C[0,1]$, для которого верна теорема Е?

Из теоремы Д вытекает, что невозможно изменением значений каждой непрерывной функции $f(x)$ на наперед заданном множестве получить функ- 
цию $g(x) \in C[0,1]$, ряд Фурье - Франклина которой абсолютно сходится равномерно на $[0,1]$. Однако если потребовать, чтобы после изменения функции $f(x) \in L^{1}[0,1]$ на заданном множестве получилась функция $g(x)$, ряд Фурье Франклина которой абсолютно сходится почти всюду на $[0,1]$, а сама функция только суммируема, то такая задача имеет положительное решение. Более того, в [8] доказана

Теорема F. Для любого $0<\epsilon<1$ существует измеримое множество $E \subset[0,1]$ с мерой $|E|>1-\epsilon$ такое, что для каждой функции $f \in L^{1}[0,1)$ можно найти функцию $\tilde{f} \in L^{1}[0,1)$, совпадающую $c f$ на $E$ и такую, что ее ряд Фурье - Франклина абсолютно сходится к ней почти всюду на $[0,1]$ и последовательность коэффициентов Фурье вновь полученной функции по системе Франклина $\left\{f_{n}(x)\right\}_{n=0}^{\infty}$ лежит во всех $l^{r}, r>2$, т. е.

$$
\sum_{n=0}^{\infty}\left|c_{n}(\tilde{f})\right|^{r}<\infty \forall r>2, \quad \text { где } c_{n}(\tilde{f})=\int_{0}^{1} \tilde{f}(x) f_{n}(x) d x .
$$

В данной статье рассмотрен вопрос о том, можно ли получить аналогичные результаты для двойных рядов Франклина.

Пусть $T=[0,1]^{2}, p \in[1, \infty)$ и $f \in L^{p}(T)$. Коэффициенты Фурье функции $f \in L^{p}(T)$ по двойной системе Франклина $\left\{f_{k}(x) f_{s}(y)\right\}_{k, s=0}^{\infty}$ обозначим через

$$
c_{k, s}(f)=\iint_{T} f(t, \tau) f_{k}(t) f_{s}(\tau) d t d \tau, \quad k, s \in \mathbb{N} \cup\{0\} .
$$

Положим

$$
\Lambda(f):=\operatorname{spec}\left\{c_{k, s}(f)\right\}=\operatorname{spec}(f)=\left\{(k, s), c_{k, s}(f)\right\} \neq 0, \quad k, s \in \mathbb{N} \cup\{0\} .
$$

Прямоугольные и сферические частичные суммы двойного ряда Фурье Франклина определяются соответственно следующим образом:

$$
\begin{aligned}
S_{N, M}(x, y, f) & :=\sum_{k=0}^{N} \sum_{s=0}^{M} c_{k, s}(f) f_{k}(x) f_{s}(y), \\
S_{R}(x, y, f) & :=\sum_{k^{2}+s^{2} \leq R^{2}} c_{k, s}(f) f_{k}(x) f_{s}(y) .
\end{aligned}
$$

Отметим, что ряд результатов невозможно перенести с одномерного случая на двумерный случай. В этом случае даже разные (сферические, прямоугольные, квадратные) частичные суммы резко отличаются друг от друга по своим свойствам в таких вопросах, как сходимость в $L^{p}, p \geq 1$, и сходимость почти всюду.

Подтверждением изложенного выше служит, в частности, следующий результат: существует суммируемая на $T$ функция $f_{0}(x, y)$, прямоугольные частичные суммы двойного ряда Фурье - Хаара [10] которой расходятся почти всюду на $T$ (нам не известно, верен ли такой результат для двойного ряда Фурье - Франклина).

Заметим, что в одномерном случае картина такова: ряд Фурье - Франклина каждой функции $f \in L^{1}[0,1]$ сходится почти всюду на $[0,1]$.

Естествен вопрос: существует ли измеримое множество $e$ сколь угодно малой меры такое, что после изменения значений любой функции $g \in L^{1}(T)$ на $e$ ряд Фурье по двойной системе Франклина $\left\{f_{k}(x) f_{s}(y)\right\}_{k, s=0}^{\infty}$ измененной функции сходится почти всюду на $T$ по прямоугольникам или по сферам?

Оказывается, что ответ на этот вопрос положителен. Имеет место 
Теорема 1. Для любого $0<\epsilon<1$ существует измеримое множество $E \subset T=[0,1]^{2}$ с мерой $|E|>1-\epsilon$ такое, что для каждой функции $f \in L^{1}(T)$ можно найти функцию $\tilde{f} \in L^{1}(T)$, совпадающую с $f(x, y)$ на $E$ и такую, что как прямоугольные, так и сферические частичные суммы ее двойного ряда Фурье Франклина сходятся к ней почти всюду на $T$.

Более того, в настоящей статье доказывается

Теорема 2. Для любого $0<\epsilon<1$ существует измеримое множество $E \subset$ $T=[0,1]^{2}$ с мерой $|E|>1-\epsilon$ такое, что для каждой функции $f \in L^{1}(T)$ и для любого $0<\eta<1$ можно найти функцию $\tilde{f} \in L^{1}(T) c \iint_{T}|f(x, y)-\tilde{f}(x, y)| d x d y \leq$ $\eta$, совпадающую с $f(x, y)$ на $E$ и такую, что ее двойной ряд Фурье - Франклина абсолютно сходится к ней почти всюду на $T$.

Эта теорема следует из следующей более сильной теоремы.

Теорема 3. Существует ряд по двойной системе Франклина вида

$$
\sum_{k=0}^{\infty} \sum_{s=0}^{\infty} d_{k, s} f_{k}(x) f_{s}(y), \quad \sum_{k, s=0}^{\infty}\left|d_{k, s}\right|^{r}<\infty, \quad r>2,
$$

со свойством: для любого $0<\epsilon<1$ существует измеримое множество $E \subset T$ c мерой $|E|>1-\epsilon$ такое, что для каждой функции $f \in L^{1}(T)$ и для любого $0<\eta<1$ можно найти функцию $\tilde{f} \in L^{1}(T) c \iint_{T}|f(x, y)-\tilde{f}(x, y)| d x d y \leq \eta$, совпадающую с $f(x, y)$ на $E$ и такую, что ее ряд Фурье - Франклина абсолютно сходится к ней почти всюду на $T$ и

$$
c_{k, s}(\tilde{f})=d_{k, s}, \quad(k, s) \in \Lambda(\tilde{f})=\operatorname{spec}(\tilde{f}) .
$$

Вопрос 2. Возможно ли в качестве ряда (1.5) выбрать ряд Фурье по двойной системе Франклина некоторой функции $g \in L^{1}(T)$ ?

\section{$\S 2$. Доказательство основной леммы}

Напомним определение системы Франклина [3]. Пусть $\pi_{1}=\{0,1\}$ и при $n=2^{k}+i, k=0,1, \ldots, i=1,2, \ldots, 2^{k}$,

$$
\pi_{n}=\left\{t_{s}\right\}_{s=0}^{n}, \quad \text { где } t_{s}=t_{s}(n)= \begin{cases}\frac{s}{2^{k+1}}, & \text { если } s=0,1, \ldots, 2 i, \\ \frac{s-i}{2^{k}}, & \text { если } s=2 i+1, \ldots, n .\end{cases}
$$

Через $S_{n}$ обозначим пространство функций, непрерывных на $[0,1]$ и кусочно линейных с узлами из $\pi_{n}$. Отметим что $\pi_{n}$ получается добавлением в $\pi_{n-1}$ точки $z_{n}=t_{2 i-1}(n)=\frac{2 i-1}{2^{k+1}}$.

Система функций Франклина $F=\left\{f_{n}(x)\right\}$ определяется на $[0,1]$ следующим образом:

$$
f_{0}(x)=1, \quad f_{1}(x)=\sqrt{3}(2 x-1), \quad x \in[0,1],
$$

и при $n \geq 2$

$$
f_{n}(x) \in S_{n}, \quad f_{n} \perp S_{n-1}, \quad\left\|f_{n}\right\|_{L^{2}}=1, \quad f_{n}\left(t_{2 i-1}(n)\right)>0 .
$$

Разобьем отрезок $[0,1]$ на $2^{q}$ равных частей: $\Delta_{q}^{(j)}=\left[\frac{j-1}{2^{q}}, \frac{j}{2^{q}}\right], 1 \leq i \leq 2^{q}$, которые будем называть двоичными отрезками. 
ЗАмЕчАниЕ 1. Для каждой функции $f \in L^{1}(T)$ и для любого положительного числа $\xi$ существует полином $Q(x, y)$ по двойной системе Франклина $\left\{f_{k}(x) f_{s}(y)\right\}_{k, s=0}^{\infty}$ со свойством

$$
\frac{3}{4} \xi \leq \iint_{T}|f(x, y)-Q(x, y)| d x d y \leq \frac{5}{4} \xi .
$$

Действительно, легко видеть, что можно выбрать полином $Q(x, y)$ по двойной системе Франклина $\left\{f_{k}(x) f_{s}(y)\right\}_{k, s=0}^{\infty}$ такой, что

$$
\iint_{T}|(f(x, y)-\xi)-Q(x, y)| d x d y \leq \frac{\xi}{4} .
$$

Отсюда вытекает, что

$$
\begin{gathered}
\iint_{T}|f(x, y)-Q(x, y)| d x d y \leq \frac{\xi}{4}+\xi \\
\iint_{T}|f(x, y)-Q(x, y)| d x d y \geq \iint_{T} \xi d x d y-\iint_{T}|[f(x, y)-\xi]-Q(x, y)| d x d y \geq \xi-\frac{\xi}{4} .
\end{gathered}
$$

Лемма 1. Для каждой функции $f \in L^{1}(T)$ и для любой последовательности положительных чисел $\left\{\xi_{k}\right\}_{k=1}^{\infty} c$

$$
0<\xi_{k+1} \leq \frac{\xi_{k}}{5}, \quad k \geq 1,
$$

можно найти последовательность полиномов $\left\{\Pi_{k}(x, y)\right\}_{k=1}^{\infty}$ по двойной системе Франклина $\left\{f_{n}(x) f_{s}(y)\right\}_{n, s=0}^{\infty}$ c рациональными коэффициентами такую, что

$$
\begin{gathered}
\lim _{N \rightarrow \infty} \iint_{T}\left|\sum_{k=1}^{N} \Pi_{k}(x, y)-f(x, y)\right| d x d y=0, \lim _{N \rightarrow \infty} \sum_{k=1}^{N} \Pi_{k}(x, y)=f(x, y) \text { п. в. на } T, \\
\frac{\xi_{k}}{2} \leq \iint_{T}\left|\Pi_{k}(x, y)\right| d x d y \leq \frac{3}{2} \xi_{k}, \quad k \geq 2 .
\end{gathered}
$$

ДокАЗАтельство. Нетрудно видеть, что в силу замечания 1 можно выбрать последовательность полиномов $\left\{Q_{k}(x, y)\right\}_{k=1}^{\infty}$ по двойной системе Франклина $\left\{f_{n}(x) f_{s}(y)\right\}_{n, s=0}^{\infty}$ с рациональными коэффициентами такую, что

$$
\frac{3}{4} \xi_{k+1} \leq \iint_{T}\left|f(x, y)-Q_{k}(x, y)\right| d x d y \leq \frac{5}{4} \xi_{k+1}, \quad k \geq 1 .
$$

Положим

$$
\Pi_{k}(x, y)=Q_{k}(x, y)-Q_{k-1}(x, y), \quad k \geq 1, Q_{0}(x, y)=0 .
$$

Из (2.3) и (2.4) вытекает, что

$$
\begin{aligned}
\iint_{T}\left|f(x, y)-\sum_{k=1}^{N} \Pi_{k}(x, y)\right| & d x d y \\
& =\iint_{T}\left|f(x, y)-Q_{N}(x, y)\right| d x d y \leq \frac{5}{4} \xi_{N}, \quad N \geq 1 .
\end{aligned}
$$


В силу (2.2)-(2.4) получим

$$
\begin{gathered}
\iint_{T}\left|\Pi_{k}(x, y)\right| d x d y \leq \iint_{T}\left|f(x, y)-Q_{k}(x, y)\right| d x d y \\
\quad+\iint_{T}\left|f(x, y)-Q_{k-1}(x, y)\right| d x d y \leq \frac{3}{2} \xi_{k}, \quad k \geq 2, \\
\iint_{T}\left|\Pi_{k}(x, y)\right| d x d y \geq \iint_{T}\left|f(x, y)-Q_{k-1}(x, y)\right| d x d y \\
-\iint_{T}\left|f(x, y)-Q_{k}(x, y)\right| d x d y \geq \frac{\xi_{k}}{2}, \quad k \geq 2 .
\end{gathered}
$$

Полагая

$$
B:=\bigcup_{q=1}^{\infty} \bigcap_{N=q}^{\infty}\left\{(x, y) \in T:\left|f(x, y)-\sum_{k=1}^{N} \Pi_{k}(x, y)\right|<\sqrt{\xi_{N}}\right\}
$$

и учитывая соотношения $(2.2),(2.5)$, будем иметь $|B|=1$.

Ясно, что на множестве $B$ имеет место $\left|f(x, y)-\sum_{k=1}^{N} \Pi_{k}(x, y)\right| \rightarrow 0$ при $\rightarrow \infty$, следовательно,

Лемма 1 доказана.

$$
\lim _{N \rightarrow \infty} \sum_{k=1}^{N} \Pi_{k}(x, y)=f(x, y) \quad \text { п. в. на } T .
$$

Ниже будем использовать следующую лемму, доказанную в [8, лемма 6].

Лемма 2. Пусть заданы числа $\varepsilon_{0}, \delta_{0}, \lambda_{0}, \theta_{0}, \tau_{0}, \sigma_{0} \in(0,1), N_{0} \in \mathbb{N},\left(\varepsilon_{0}<\delta_{0}\right)$ и двоичный отрезок $\Delta=[a, b]$. Тогда существуют полином по системе Франклина вида $Q(t)=\sum_{n=N_{0}}^{M} a_{n} f_{n}(t)$ и множества $G \subset E \subset[a, b]$ такие, что

$$
\begin{gathered}
|E|>\left(1-\varepsilon_{0}\right)(b-a), \quad|G|>\left(1-\delta_{0}\right)(b-a), \\
Q(t)=0 \quad \text { при всех } t \notin[a, b], \\
Q(t)=1 \quad \text { при всех } x \in E, \\
\int_{[a, b]}|Q(t)| d t<2(b-a), \\
\sum_{n=N}^{M}\left|a_{n} f_{n}(t)\right|<\theta_{0} \quad \text { для всех } t \notin\left(a-\lambda_{0}, b+\lambda_{0}\right), \\
\left(\sum_{n=N_{0}}^{M}\left|a_{n}\right|^{2+\sigma_{0}}\right)^{\frac{1}{2+\sigma_{0}}}<\tau_{0}, \\
\sum_{n=N_{0}}^{M}\left|a_{n} f_{n}(t)\right|<\frac{A\left(\ln \frac{1}{\delta_{0}}\right)}{\delta_{0}} \text { для всех } t \in G, \text { где } A-\text { постоянная. }
\end{gathered}
$$


Лемма 3. Пусть заданы числа $0<\eta<\varepsilon<\delta<1, r>2, N \in \mathbb{N}$ и функция $f(x, y) \in L^{1}(T) c \iint_{T}|f(x, y)| d x d y>0$. Тогда существуют множества $G \subset E \subset T$, функция $g(x, y) \in L^{1}(T)$ и полином по двоиной системе Франклина вида

$$
Q(x, y)=\sum_{k, s=N}^{M} c_{k, s} f_{k}(x) f_{s}(y)
$$

такие, что

$$
\begin{gathered}
|E|>1-\varepsilon, \quad|G|>1-\delta, \\
g(x, y)=f(x, y) \quad \text { на } E, \\
\iint_{T}|g(x, y)-Q(x, y)| d x d y \leq \eta, \\
\iint_{T}|g(x, y)| d x d y \leq 5 \iint_{T}|f(x, y)| d x d y,
\end{gathered}
$$

$$
\begin{aligned}
& \sum_{k, s=N}^{M}\left|c_{k, s} f_{n}(x) f_{s}(y)\right| \leq \frac{B\left(\ln \frac{1}{\delta}\right)^{2}|f(x, y)|}{\delta^{2}}+\eta, \quad(x, y) \in G, \text { где } B-\text { постоянная, } \\
& \left(\sum_{k, s=N}^{M}\left|c_{k, s}\right|^{r}\right)^{\frac{1}{r}} \leq \eta
\end{aligned}
$$

ДоКАЗАТЕЛЬСТво ЛЕмМЫ 3. Возьмем ступенчатую функцию вида

$$
\varphi(x, y)=\sum_{l, j=1}^{2^{q}} \gamma_{l, j} \chi_{\Delta_{l, j}}(x, y)
$$

где

$$
\begin{gathered}
\Delta_{l, j}=\Delta_{l}^{\prime} \times \Delta_{j}^{\prime \prime}=\left[\alpha_{l-1}, \alpha_{l}\right] \times\left[\alpha_{j-1}, \alpha_{j}\right], \\
\alpha_{j}=\frac{j}{2^{q}}, \quad j=0,1, \ldots, 2^{q},
\end{gathered}
$$

такую, что

$$
\iint_{T}|f(x, y)-\varphi(x, y)| d x d y<\min \left[\frac{\eta \delta^{3}}{128 A^{2}\left(\ln \frac{1}{\delta}\right)^{2}} ; \frac{1}{2} \iint_{T}|f(x, y)| d x d y\right] .
$$

Пусть

$$
E_{0}=\left\{(x, y) \in T:|f(x, y)-\varphi(x, y)|<\frac{\eta \delta^{2}}{32 A^{2}\left(\ln \frac{1}{\delta}\right)^{2}}\right\} .
$$

В силу (2.9) и (2.10) получим

$$
\left|E_{0}\right|>\left(1-\frac{\delta}{4}\right)
$$


Положим

$$
\partial=\frac{\delta \eta}{\left(4 A \sum_{l, j=1}^{2^{q}}\left|\gamma_{l, j}\right|+1\right)\left(\ln \frac{1}{\delta}\right)}
$$

Применим лемму 2 (для каждого $l \in\left[1,2^{q}\right]$ ), полагая в ее формулировке $\Delta=\Delta_{l}^{\prime}, \varepsilon_{0}=\frac{\varepsilon}{4}, \delta_{0}=\frac{\delta}{4}, N_{0}=N_{l}, \tau_{0}=\left(\sum_{l, j=1}^{2^{q}}\left|\gamma_{l, j}\right|\right)^{-1}, \sigma_{0}=r-2, \lambda_{0}=\frac{\delta}{2^{q+4}}$, $\theta_{0}=\partial$. Тогда определяются измеримые множества $G_{l}^{\prime} \subset E_{l}^{\prime} \subset \Delta_{l}^{\prime}$ и полиномы по системе Франклина вида

$$
Q_{l}^{\prime}(x)=\sum_{n=N_{l}+1}^{N_{l+1}} a_{k}^{(l)} f_{n}(x)
$$

для каждого $l \in\left[1,2^{q}\right]$, удовлетворяющие условиям

$$
\begin{gathered}
\left|E_{l}^{\prime}\right|>\left|\Delta_{l}^{\prime}\right|\left(1-\frac{\varepsilon}{4}\right), \quad\left|G_{l}^{\prime}\right|>\left|\Delta_{l}^{\prime}\right|\left(1-\frac{\delta}{4}\right), \\
Q_{l}^{\prime}(x)= \begin{cases}1 & \text { при } x \in E_{l}^{\prime}, \\
0 & \text { при } x \notin \Delta_{l}^{\prime},\end{cases} \\
\int_{\Delta_{l}^{\prime}}\left|Q_{l}(x)\right| d x<2\left|\Delta_{l}^{\prime}\right|, \\
\sum_{k=N_{l}+1}^{N_{l+1}}\left|a_{k}^{(l)} f_{k}(x)\right|<\partial, \quad x \notin\left[\alpha_{l}-\frac{\delta}{2^{q+4}}, \alpha_{l+1}+\frac{\delta}{2^{q+4}}\right], \\
\sum_{k=N_{l}+1}^{N_{l+1}}\left|a_{k}^{(l)} f_{k}(x)\right|<\frac{4 A\left(\ln \frac{1}{\delta}\right)}{\delta}, \quad x \in G_{l}^{\prime}, \\
\left(\sum_{k=N_{l}+1}^{N_{l+1}}\left|a_{k}^{(l)}\right|^{r}\right)^{\frac{1}{r}}<\left(\sum_{l, j=1}^{2^{q}}\left|\gamma_{l, j}\right|\right)^{-1} .
\end{gathered}
$$

Снова применим лемму 2 (для каждого $j \in\left[1,2^{q}\right]$ ), полагая в ее формулировке

$$
\Delta=\Delta_{j}^{\prime \prime}, \varepsilon_{0}=\frac{\varepsilon}{4}, \delta_{0}=\frac{\delta}{4}, N_{0}=N_{2^{q}+1}, \tau_{0}=\eta, \sigma_{0}=r-2, \lambda_{0}=\frac{\delta}{2^{q+4}}, \theta_{0}=\partial .
$$

Тогда определяются измеримые множества $G_{j}^{\prime \prime} \subset E_{j}^{\prime \prime} \subset \Delta_{j}^{\prime \prime}$ и полином по системе Франклина вида

$$
Q_{j}^{\prime \prime}(y)=\sum_{s=M_{j}+1}^{M_{j+1}} b_{s}^{(j)} f_{s}(y), \quad M_{1}=N_{2^{q}},
$$

для каждого $j \in\left[1,2^{q}\right]$, удовлетворяющие условиям

$$
\begin{gathered}
\left|E_{j}^{\prime \prime}\right|>\left|\Delta_{j}^{\prime \prime}\right|\left(1-\frac{\varepsilon}{4}\right), \quad\left|G_{j}^{\prime \prime}\right|>\left|\Delta_{j}^{\prime \prime}\right|\left(1-\frac{\delta}{4}\right), \\
Q_{j}^{\prime \prime}(y)= \begin{cases}1 & \text { при } y \in E_{j}^{\prime \prime}, \\
0 & \text { при } x \notin \Delta_{j}^{\prime \prime},\end{cases}
\end{gathered}
$$




$$
\begin{gathered}
\int_{\Delta_{j}^{\prime \prime}}\left|Q_{j}^{\prime \prime}(y)\right| d y<2\left|\Delta_{j}^{\prime \prime}\right|, \\
\sum_{s=M_{j}+1}^{M_{j+1}}\left|b_{s}^{(j)} f_{s}(y)\right|<\partial, \quad y \notin\left[\beta_{j}-\frac{\delta}{2^{q+4}}, \beta_{j+1}+\frac{\delta}{2^{q+4}}\right], \\
\sum_{s=M_{j}+1}^{M_{j+1}}\left|b_{s}^{(j)} f_{s}(y)\right|<\frac{A\left(\ln \frac{1}{\delta}\right)}{\delta}, \quad x \in G_{j}^{\prime \prime}, \\
\left(\sum_{s=M_{j}+1}^{M_{j+1}}\left|b_{s}^{(j)}\right|^{r}\right)^{\frac{1}{r}}<\eta .
\end{gathered}
$$

Определим полином $Q(x, y)$, функцию $g(x, y)$ и множества $E, G$ следующим образом:

$$
\begin{array}{r}
Q(x, y)=\sum_{l, j=1}^{2^{q}} \gamma_{l, j} Q_{l}^{\prime}(x) Q_{j}^{\prime \prime}(y)=\sum_{l, j=1}^{2^{q}} \gamma_{l, j} \sum_{k=N_{l}+1}^{N_{l+1}} a_{k}^{(l)} f_{k}(x) \sum_{s=M_{j}+1}^{M_{j+1}} b_{s}^{(j)} f_{s}(y) \\
=\sum_{k, s=N}^{M} c_{k, s} f_{k}(x) f_{s}(y), \quad M=M_{2^{q}+1},
\end{array}
$$

где

$$
\begin{gathered}
c_{k, s}= \begin{cases}\gamma_{l, j} a_{k}^{(l)} b_{s}^{(j)}, & (k, s) \in \Omega_{l, j}:=\left(N_{l}, N_{l+1}\right] \times\left(M_{j}, M_{j+1}\right], l, j \in\left[1,2^{q}\right], \\
0, & (k, s) \notin \bigcup_{l j=1}^{2^{q}} \Omega_{l, j}\end{cases} \\
g(x, y)=f(x, y)-[\varphi(x, y)-Q(x, y)], \\
E=\bigcup_{l=1}^{2^{q} 2^{q}} \bigcup_{j=1}^{2_{l}}\left(E_{l}^{\prime} \times E_{j}^{\prime \prime}\right), \\
G=E_{0} \cap\left[\left(\bigcup_{l=1}^{2^{q}} G_{l}^{\prime}\right) \times\left(\bigcup_{j=1}^{2^{q}} G_{j}^{\prime \prime}\right)\right] \backslash E_{0} \cap\left[\left(A_{q} \times[0,1]\right) \cup\left([0,1] \times A_{q}\right)\right]
\end{gathered}
$$

где $A_{q}=\bigcup_{l=1}^{2^{q}}\left[\alpha_{j}-\frac{\delta}{2^{q+4}}, \alpha_{j}+\frac{\delta}{2^{q+4}}\right]$. Из условий $(2.6),(2.11),(2.14),(2.15),(2.21)$, (2.22), (2.27), (2.29)-(2.31) следует, что

$$
|E|>1-\varepsilon,|G|>1-\delta, g(x, y)=f(x, y) \text { на } E, \iint_{T}|g(x, y)-Q(x, y)| d x d y \leq \eta \text {. }
$$

В силу (2.6), (2.15), (2.16), (2.22), (2.23), (2.27) имеем

$$
\begin{aligned}
\iint_{T}|Q(x, y)| d x d y=\sum_{l, j=1}^{q}\left|\gamma_{l, j}\right| \int_{\Delta_{l}^{\prime}} \mid & Q_{l}(x)\left|d x \int_{\Delta_{j}^{\prime \prime}}\right| Q_{j}^{\prime \prime}(y) \mid d y \\
& \leq 4 \sum_{l, j=1}^{q}\left|\gamma_{l, j}\right|\left|\Delta_{l}^{\prime}\right|\left|\Delta_{j}^{\prime \prime}\right|=4 \iint_{T}|\varphi(x, y)| d x d y .
\end{aligned}
$$


Отсюда и из $(2.9),(2.29)$ вытекает, что

$$
\begin{aligned}
\iint_{T}|g(x, y)| d x d y \leq \iint_{T} \mid f(x, y)- & \varphi(x, y) \mid d x d y \\
& +\iint_{T}|Q(x, y)| d x d y \leq 5 \iint_{T}|f(x, y)| d x d y .
\end{aligned}
$$

Проверим выполнение утверждения (5) леммы 3.

Пусть $(x, y) \in G$, тогда для некоторых $l_{0}$ и $j_{0},(x, y) \in G_{l_{0}}^{\prime} \times G_{j_{0}}^{\prime \prime}$. Учитывая соотношения $(2.6),(2.12),(2.17),(2.18),(2.24),(2.25),(2.28)$ и $(2.31)$, имеем

$$
\begin{gathered}
\sum_{k, s=N}^{M}\left|c_{k, s} f_{n}(x) f_{s}(y)\right| \leq \sum_{l, j=1}^{2^{q}}\left|\gamma_{l, j}\right|\left(\sum_{n=N_{l}+1}^{N_{l+1}}\left|a_{k}^{(l)} f_{k}(x)\right|\right)\left(\sum_{s=M_{j}+1}^{M_{j+1}}\left|b_{s}^{(j)} f_{s}(y)\right|\right) \\
=\left|\gamma_{l_{0}, j_{0}}\right|\left(\sum_{n=N_{l_{0}}+1}^{N_{l_{0}+1}}\left|a_{k}^{\left(l_{0}\right)} f_{k}(x)\right|\right)\left(\sum_{s=M_{j_{0}+1}}^{M_{j_{0}+1}}\left|b_{s}^{\left(j_{0}\right)} f_{s}(y)\right|\right) \\
+\sum_{(l, j) \in\left[1,2^{q}\right]^{2} \backslash\left(l_{0}, j_{0}\right)}\left|\gamma_{l, j}\right|\left(\sum_{n=N_{l}+1}^{N_{l+1}}\left|a_{k}^{(l)} f_{k}(x)\right|\right)\left(\sum_{s=M_{j}+1}^{M_{j+1}}\left|b_{s}^{(j)} f_{s}(y)\right|\right) \\
\leq \frac{16 A^{2}\left(\ln \frac{1}{\delta}\right)^{2}\left|\gamma_{l_{0}, j_{0}}\right|}{\delta^{2}}+2\left(\sum_{l, j=1}^{2^{q}}\left|\gamma_{l, j}\right|\right)\left(\frac{A \ln \frac{1}{\delta}}{\delta} \partial+\partial^{2}\right) \\
\leq \frac{B\left(\ln \frac{1}{\delta}\right)^{2}|\varphi(x, y)|}{\delta^{2}}+\frac{\eta}{2}, \quad \text { где } B=16 A^{2} .
\end{gathered}
$$

Воспользовавшись неравенством (см. (2.9), (2.31))

$$
|\varphi(x, y)|<|f(x, y)|+\frac{\eta \delta^{2}}{32 A^{2}\left(\ln \frac{1}{\delta}\right)^{2}}, \quad(x, y) \in G \subset E_{0},
$$

из (2.32) получим

$$
\sum_{k, s=N}^{M}\left|c_{k, s} f_{k}(x) f_{s}(y)\right| \leq \frac{B\left(\ln \frac{1}{\delta}\right)^{2}|f(x, y)|}{\delta^{2}}+\eta .
$$

В силу $(2.19),(2.26)$ и $(2.28)$ имеем

$$
\begin{aligned}
\left(\sum_{k, s=N}^{M}\left|c_{k, s}\right|^{r}\right)^{\frac{1}{r}} & =\left(\sum_{l, j=1}^{2^{q}} \sum_{n=N_{l}+1}^{N_{l+1}} \sum_{s=M_{j}+1}^{M_{j+1}}\left|\gamma_{l, j} a_{k}^{(l)} b_{s}^{(j)}\right|^{r}\right)^{\frac{1}{r}} \\
& =\left(\sum_{l, j=1}^{2^{q}}\left|\gamma_{l, j}\right|^{r} \sum_{n=N_{l}+1}^{N_{l+1}}\left|a_{k}^{(l)}\right|^{r} \sum_{s=M_{j}+1}^{M_{j+1}}\left|b_{s}^{(j)}\right|^{r}\right)^{\frac{1}{r}} \\
& \leq \sum_{l, j=1}^{2^{q}}\left|\gamma_{l, j}\right|\left(\sum_{k=N}^{M}\left|a_{k}^{(l)}\right|^{r}\right)^{\frac{1}{r}}\left(\sum_{s=N}^{M}\left|b_{k}^{(j)}\right|^{r}\right)^{\frac{1}{r}} \leq \eta .
\end{aligned}
$$

Лемма 3 доказана. 


\section{§ 3. Доказательство теоремы 3}

Пусть $\epsilon>0$. Обозначим через

$$
\left\{\phi_{n}(x, y)\right\}_{n=1}^{\infty}
$$

последовательность полиномов по двойной системе Франклина $\left\{f_{k}(x) f_{s}(y)\right\}_{k, s=0}^{\infty}$ с рациональными коэффициентами и положим

$$
\delta_{n}=\min \left\{\frac{1}{2} ; \sqrt[4]{\iint_{T}\left|\phi_{n}(x, y)\right| d x d y}\right\} .
$$

Применяя лемму 3 , можно найти последовательности множеств $\left\{G_{n}\right\},\left\{E_{n}\right\}$, функций $\left\{g_{n}(x, y)\right\}$ и полиномов по двоиной системе Франклина вида

$$
Q_{n}(x, y)=\sum_{k, s=M_{n}+1}^{M_{n+1}} c_{k, s}^{(n)} f_{k}(x) f_{s}(y),
$$

которые для всех $n \geq 1$ удовлетворяют условиям

$$
\begin{gathered}
E_{n}, G_{n} \subset T, \quad\left|E_{n}\right|>1-\epsilon 2^{-(n+2)}, \quad\left|G_{n}\right|>1-\delta_{n}, \\
g_{n}(x, y)=\phi_{n}(x, y) \quad \text { на } E_{n}, \\
\iint_{T}\left|g_{n}(x, y)-Q_{n}(x, y)\right| d x d y \leq 2^{-5(n+3)}, \\
\iint_{T}\left|g_{n}(x, y)\right| d x d y \leq 5 \iint_{T}\left|\phi_{n}(x, y)\right| d x d y, \\
\sum_{k, s=M_{n}+1}^{M_{n+1}}\left|c_{k, s}^{(n)} f_{k}(x) f_{s}(y)\right| \leq \frac{B\left(\ln \frac{1}{\delta_{n}}\right)^{2}\left|\phi_{n}(x, y)\right|}{\delta_{n}^{2}}+2^{-5(n+3)}, \quad(x, y) \in G_{n}, \\
\left(\sum_{k, s=M_{n}+1}^{M_{n+1}}\left|c_{k, s}^{(n)}\right|^{2+2^{-n}}\right)^{\frac{1}{2+2^{-n}}} \leq 2^{-5(n+3)} .
\end{gathered}
$$

Положим

$$
\begin{gathered}
E=\bigcap_{n=1}^{\infty} E_{n}, \\
\sum_{k, s=0}^{\infty} d_{k, s} f_{k}(x) f_{s}(y)=\sum_{n=1}^{\infty} Q_{n}(x, y)=\sum_{n=1}^{\infty} \sum_{k, s=M_{n}+1}^{M_{n+1}} c_{k, s}^{(n)} f_{k}(x) f_{s}(y), \\
d_{k, s}= \begin{cases}c_{k, s}^{(n)} & \text { при }(k, s) \in \Omega:=\bigcup_{n=1}^{\infty}\left(M_{n} ; M_{n+1}\right] \times\left(M_{n} ; M_{n+1}\right], \\
0 & \text { при }(k, s) \notin \Omega .\end{cases}
\end{gathered}
$$

Очевидно, что (см. (3.4), (3.9), (3.10) и (3.12))

$$
|E|>1-\epsilon, \quad \sum_{k, s=0}^{\infty}\left|d_{k, s}\right|^{r}<\infty, r>2 .
$$


Пусть $f(x, y) \in L^{1}(T)$ и $\eta-$ любое положительное число. В силу леммы 1 можно выбрать подпоследовательность $\left\{\phi_{n_{k}}(x, y)\right\}_{n=1}^{\infty}$ из последовательности (3.1) такую, что

$$
\begin{gathered}
\lim _{N \rightarrow \infty} \iint_{T}\left|\sum_{k=1}^{N} \phi_{n_{k}}(x, y)-f(x, y)\right| d x d y=0, \\
\lim _{N \rightarrow \infty} \sum_{k=1}^{N} \phi_{n_{k}}(x, y)=f(x, y) \quad \text { п. в. на } T, \\
\eta 2^{-4(k+1)} \leq \iint_{T}\left|\phi_{n_{k}}(x, y)\right| d x d y \leq 3 \eta 2^{-4(k+1)}, \quad k \geq 2 .
\end{gathered}
$$

Из (3.13) и (3.15) получим

$$
\iint_{T}\left|f(x, y)-\phi_{n_{1}}(x, y)\right| d x d y<\frac{\eta}{2} .
$$

Предположим, что уже определены числа $n_{1}=\nu_{1}<\cdots<\nu_{q-1}$, функции $g_{1}(x, y)=\phi_{n_{1}}(x, y), g_{2}(x, y), \ldots, g_{q-1}(x, y)$ и полиномы

$$
Q_{\nu_{n}}(x, y)=\sum_{k, s=M_{\nu_{n}}+1}^{M_{\nu_{n}+1}} c_{k, s}^{\left(\nu_{n}\right)} f_{k}(x) f_{s}(y), \quad 1 \leq n \leq q-1,
$$

удовлетворяющие следующим условиям:

$$
\begin{gathered}
g_{l}(x, y)=\phi_{k_{l}}(x, y), \quad(x, y) \in E, \quad l \in[1, q-1] \\
\iint_{T}\left|g_{l}(x, y)\right| d x d y<2^{-(l+1)}, \quad l \in[1, q-1] \\
\iint_{T}\left|\sum_{j=1}^{l}\left[g_{j}(x, y)-Q_{\nu_{j}}(x, y)\right]\right| d x d y<\eta 2^{-5(l+2)}, \quad l \in[1, q-1] .
\end{gathered}
$$

Легко видеть, что можно выбрать натуральное число $\nu_{q}>\nu_{q-1}$ (функцию $\phi_{\nu_{q}}(x, y)$ из последовательности (3.1)) таким образом, что

$\iint_{T}\left|\left\{\phi_{n_{q}}(x, y)-\sum_{j=1}^{q-1}\left[g_{j}(x, y)-Q_{\nu_{j}}(x, y)\right]\right\}-\phi_{\nu_{q}}(x, y)\right| d x d y \leq \eta 2^{-6(q+3)}$.

Покажем, что

$$
\eta 2^{-4 q-5} \leq \iint_{T}\left|\phi_{\nu_{q}}(x, y)\right| d x d y \leq \eta 2^{-4 q} .
$$

В силу (3.15), (3.17) и (3.18) имеем

$$
\begin{gathered}
\iint_{T}\left|\phi_{\nu_{q}}(x, y)\right| d x d y \geq \iint_{T}\left|\phi_{n_{q}}(x, y)\right| d x d y-\iint_{T}\left|\sum_{j=1}^{q-1}\left[g_{j}(x, y)-Q_{\nu_{j}}(x, y)\right]\right| d x d y \\
-\iint_{T}\left|\left\{\phi_{n_{q}}(x, y)-\sum_{j=1}^{q-1}\left[g_{j}(x, y)-Q_{\nu_{j}}(x, y)\right]\right\}-\phi_{\nu_{q}}(x, y)\right| d x d y
\end{gathered}
$$




$$
\geq \eta 2^{-4(q+1)}-\eta 2^{-5(q+2)}-\eta 2^{-6(q+3)} \geq \eta 2^{-4 q-5}
$$

и аналогично

$$
\iint_{T}\left|\phi_{\nu_{q}}(x, y)\right| d x d y \leq \eta 2^{-4(q+1)}+\eta 2^{-5(q+2)}+\eta 2^{-6(q+3)} \leq \eta 2^{-4 q} .
$$

Положим

$$
g_{q}(x, y)=\phi_{n_{q}}(x, y)+\left[g_{\nu q}(x, y)-\phi_{\nu_{q}}(x, y)\right]
$$

Отсюда и из (3.5), (3.10) вытекает, что

$$
g_{q}(x, y)=\phi_{n_{q}}(x, y), \quad(x, y) \in E, q \geq 1 .
$$

Учитывая соотношения (3.6), (2.18) и (3.20), для всех $q \geq 2$ будем иметь

$$
\begin{aligned}
\iint_{T} \mid \sum_{j=1}^{q} & {\left[g_{j}(x, y)-Q_{\nu_{j}}(x, y)\right] \mid d x d y } \\
\leq \iint_{T} \mid\left\{\phi_{n_{q}}(x, y)\right. & \left.-\sum_{j=1}^{q-1}\left[g_{j}(x, y)-Q_{\nu_{j}}(x, y)\right]\right\}-\phi_{\nu_{q}}(x, y) \mid d x d y \\
& +\iint_{T}\left|g_{\nu_{q}}(x, y)-Q_{\nu_{q}}(x, y)\right| d x d y \leq \eta 2^{-5(q+2)} .
\end{aligned}
$$

В силу (3.15), (3.19) и (3.20) получим

$$
\begin{aligned}
\iint_{T}\left|g_{q}(x, y)\right| d x d y \leq & \iint_{T}\left|\phi_{n_{q}}(x, y)\right| d x d y \\
& +\iint_{T}\left|g_{\nu_{q}}(x, y)\right| d x d y+\iint_{T}\left|\phi_{\nu_{q}}(x, y)\right| d x d y \leq \eta 2^{-q} .
\end{aligned}
$$

Ясно, что по индукции определяются последовательности функций $\left\{g_{q}(x, y)\right\}_{q=1}^{\infty}$ $\left(g_{1}(x, y)=\phi_{n_{1}}(x, y)\right)$ и полиномов $\left\{Q_{\nu_{q}}(x, y\}\right.$, удовлетворяющих условиям (3.21)(3.23) для всех $q>1$. Из (3.23) вытекает, что

$$
\sum_{q=1}^{\infty} \iint_{T}\left|g_{q}(x, y)\right| d x d y<\infty
$$

Определим функцию $\tilde{f}(x, y)$ и последовательность чисел $\left\{\varepsilon_{k, s}\right\}_{k, s=0}^{\infty}$ следующим образом:

$$
\begin{gathered}
\tilde{f}(x, y)=\sum_{q=1}^{\infty} g_{q}(x, y), \\
\varepsilon_{k, s}= \begin{cases}1 & \text { при }(k, s) \in \Omega_{0}:=\bigcup_{q=1}^{\infty}\left(M_{\nu_{q}}, M_{\nu_{q}+1}\right] \times\left(M_{\nu_{q}}, M_{\nu_{q}+1}\right], \\
0 & \text { при }(k, s) \notin \Omega_{0} .\end{cases}
\end{gathered}
$$

Учитывая соотношения (3.14), (3.21) и (3.24), получим $\tilde{f} \in L^{1}(T), \tilde{f}(x, y)=$ $f(x, y),(x, y) \in E$. Поскольку $g_{1}(x, y)=\phi_{n_{1}}(x, y)$, из $(3.13),(3.16)$ и $(3.24)$ будем иметь

$$
\begin{aligned}
& \iint_{T}|f(x, y)-\tilde{f}(x, y)| d x d y \leq \iint_{T}\left|f(x, y)-\phi_{n_{1}}(x, y)\right| d x d y \\
&+\sum_{q=2}^{\infty} \iint_{T}\left|g_{q}(x, y)\right| d x d y \leq \eta .
\end{aligned}
$$


Из (3.3), (3.12), (3.23)-(3.25) для всех $q \geq 2$ следует, что

$$
\begin{gathered}
\iint_{T}\left|\sum_{k, s=0}^{M_{\nu_{q}+1}} \varepsilon_{k, s} d_{k, s} f_{k}(x) f_{s}(y)-\tilde{f}(x, y)\right| d x d y \\
=\iint\left|\sum_{T}^{q} \sum_{j=1}^{M_{\nu_{j}+1}} c_{k, s=M_{\nu_{j}}+1}^{\left(\nu_{j}\right)} f_{k}(x) f_{s}(y)-\tilde{f}(x, y)\right| d x d y \\
\leq \iint_{T}\left|\sum_{j=1}^{q}\left[g_{j}(x, y)-Q_{\nu_{j}}(x, y)\right]\right| d x d y+\sum_{j=q+1}^{\infty} \iint_{T}\left|g_{j}(x, y)\right| d x d y \leq 2^{-q} .
\end{gathered}
$$

Отсюда

$$
\varepsilon_{k, s} d_{k, s}=c_{k, s}(\tilde{f})=\iint_{T} \tilde{f}(t, \tau) f_{k}(t) f_{s}(\tau) d t d \tau .
$$

Следовательно, из (3.24) и (3.25) вытекает, что

$$
c_{k, s}(\tilde{f})=d_{k, s}, \quad(k, s) \in \Lambda(f)=\operatorname{spec}(\tilde{f}) \subset \Omega_{0} .
$$

Покажем, что двойной ряд Фурье - Франклина

$$
\sum_{k, s=0}^{\infty} c_{k, s}(\tilde{f}) f_{k}(x) f_{s}(y)=\sum_{k, s=0}^{\infty} \varepsilon_{k, s} d_{k, s} f_{k}(x) f_{s}(y)
$$

функции $\tilde{f}(x, y)$ абсолютно сходится п. в. на $T$. Обозначая

$$
B_{q}=\left\{(x, y) \subset T=[0,1]^{2} ;\left|\phi_{\nu_{q}}(x, y)\right| d x \leq \eta 2^{-3 q}\right\}, \quad q \geq 2,
$$

будем иметь

$$
\eta 2^{-3 q}\left|T \backslash B_{q}\right| \leq \iint_{[0,1) \backslash B_{k}}\left|\phi_{\nu_{q}}(x, y)\right| d x d y \leq \eta 2^{-4 q}, \quad q \geq 2 .
$$

Следовательно, $\left|B_{q}\right|>1-2^{-q}$.

\section{Положим}

$$
B=\bigcup_{k=2}^{\infty} \bigcap_{q=k}^{\infty}\left(B_{q} \cap G_{\nu_{q}}\right) .
$$

Отсюда и из того, что $\left|B_{q} \cap G_{\nu_{q}}\right|>1-2^{-q+4}$ (см. (3.2), (3.4), (3.19) и (3.28)), вытекает, что $|B|=1$.

Пусть $(x, y) \in B$. Существует натуральное число $q_{0}$ такое, что $(x, y) \in$ $B_{q} \cap G_{\nu_{q}}, q \geq q_{0}$.

Учитывая соотношения (3.2), (3.8), (3.19) и (3.28), получим

$$
\sum_{k, s=M_{\nu_{q}}+1}^{M_{\nu_{q}+1}}\left|c_{k, s}^{\left(\nu_{q}\right)} f_{k}(x) f_{s}(y)\right| \leq \frac{B\left(\ln \frac{1}{\delta_{\nu_{q}}}\right)^{2}\left|\phi_{\nu_{q}}(x, y)\right|}{\delta_{\nu_{q}}^{2}}+2^{-\nu_{q}} \leq B \sqrt{\eta} q^{2} 2^{-q}+2^{-q} \text {. }
$$

Отсюда и из $(3.12),(3.26),(3.27),(3.29)$ и (3.30) заключаем, что п. в. на $T$

$$
\sum_{k, s=0}^{\infty}\left|c_{k, s}(\tilde{f}) f_{k}(x) f_{s}(y)\right|<\infty .
$$

Нетрудно убедиться, что (см. (3.26), (3.27) и (3.30)) п. в. на $T$

$$
\lim _{N, M \rightarrow \infty} \sum_{k, s=0}^{N, M} c_{k, s}(\tilde{f}) f_{k}(x) f_{s}(y)=\tilde{f}(x, y) .
$$

Теорема 3 доказана. 


\section{ЛИТЕРАТУРА}

1. Лузин Н. Н. К основной теореме интегрального исчисления // Мат. сб. 1912. Т. 28, № 2. C. 266-294.

2. Меньшов Д. Е. О равномерной сходимости рядов Фурье // Мат. сб. 1942. Т. 53, № 2. C. 67-96.

3. Franklin Ph. A set of continous orthogonal functions // Math. Ann. 1928. V. 100. P. 522-528.

4. Арутюнян Ф. Г. О рядах по системе Хаара // Докл. АН Арм ССР. Сер. мат. 1966. Т. 42. C. $134-140$.

5. Геворкян Г. Г. О представлении измеримых функций абсолютно сходящимися рядами по системе Франклина // Докл. АН Арм ССР. Сер. мат. 1986. Т. 83, № 1. С. 15-18.

6. Katznelson Y. On a theorem of Menshoff // Proc. Amer. Math. Soc. 1975. V. 53. P. 396-398.

7. Grigoryan M. The unconditional convergence of Fourier-Haar series // Bull. TICMI. 2014. V. 18, N 1. P. 130-140.

8. Галоян Л. Н., Григорян М. Г., Кобелян А. Х. О сходимости рядов Фурье по классическим системам // Мат. сб. 2015. Т. 206, № 7. С. 55-94.

9. Grigoryan M. G., Grigoryan T. M. On the absolute convergence of Schauder series // Adv. Theoret. Appl. Math. 2014. V. 9, N 1. P. 11-14.

10. Oniani G. G. On the divergence of multiple Fourier-Haar series // Anal. Math. 2012. V. 38. P. 227-247.

Поступила в редакиию 17 августа 2019 г.

После доработки 17 августа 2019 г.

Принята к публикачии 19 февраля 2020 г.

Геворкян Гегам Григорьевич, Григорян Мартин Геворгович

Ереванский государственный университет,

ул. А. Манукяна, 1, Ереван 0025, Армения

ggg@ysu.am, gmarting@ysu.am 